\title{
Erratum to: Genomic characterization of the first class I Newcastle disease virus isolated from the mainland of China
}

Hualei Liu $\cdot$ Fei Chen $\cdot$ Yunling Zhao

Dongxia Zheng $\cdot$ Jinming $\mathrm{Li} \cdot$ Tiangang $\mathrm{Xu} \cdot$

Lin Qi $\cdot$ Zhiliang Wang

Published online: 13 March 2010

(C) Springer Science+Business Media, LLC 2010

Erratum to: Virus Genes

DOI 10.1007/s11262-010-0452-0

Some of the information provided in Figure 2 of the online published article is not pertinent to the subject matter. The correct version of Figure 2 is shown overleaf.

The online version of the original article can be found under doi: 10.1007/s11262-010-0452-0.

H. Liu - F. Chen - Y. Zhao - D. Zheng - J. Li · T. Xu •

Z. Wang $(\bowtie)$

National Reference Laboratory for Newcastle Disease,

China Animal Health and Epidemiology Center,

Qingdao 266032, China

e-mail: zlwang111@yahoo.com.cn

L. Qi

School of Animal Science and Technology, Northeast

Agricultural University, Harbin 150030, China 


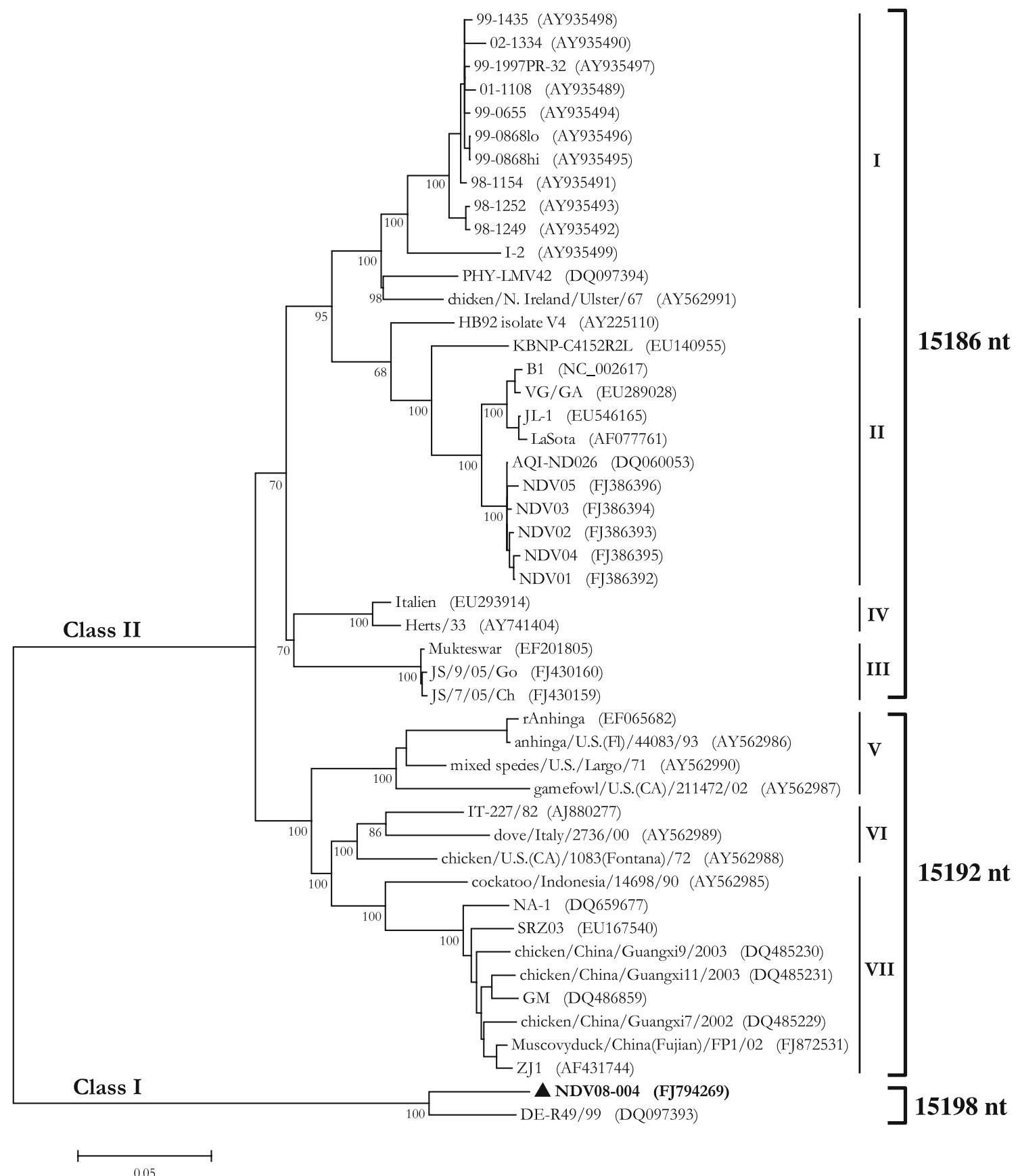

Fig. 2 Phylogenetic tree of 48 NDV strains based on the complete genomic sequence. The sequence determined in this study is in bold. The GenBank assession numbers of the NDV isolates are shown in brackets. The genomic length for different strains is indicated on the right. The tree was constructed using the neighbor-joining algorithm of MEGA 3.1, with a 1000 bootstrap trial to assign confidence to groupings 